\title{
CAMBIO CONCEPTUAL SOBRE LA COLABORACIÓN ENTRE EL PROFESORADO Y COOPERACIÓN ENTRE ESTUDIANTES
}

\author{
CONCEPTUAL CHANGE ABOUT COLLABORATION BETWEEN \\ TEACHERS AND COOPERATION BETWEEN STUDENTS
}

\section{MUDANÇA CONCEPTUAL SOBRE A COLABORAÇÃO ENTRE PROFESSORES E A COOPERAÇÃO ENTRE ESTUDANTES}

\author{
Vanessa Valdebenito Zambrano \\ Professora Doutora \\ da Universidad Católica de Temuco. Chile. \\ vvaldebenitoz@gmail.com \\ David Duran Gisbert \\ Professor Doutor \\ da Universitat Autònoma de Barcelona. Espanha. \\ david.duran@uab.cat
}

Resumen: El artículo presenta una revisión exhaustiva de la literatura sobre las potencialidades de la colaboración entre profesores en el desarrollo profesional, la resolución de problemáticas y la retroalimentación de las prácticas educativas, creando espacios de reflexión que gatillan un cambio en los enfoques, representaciones y prácticas. Después describe una red de colaboración de 13 profesores pertenecientes a 5 centros educativos, en un contexto chileno (Región de la Araucanía, Chile) de alta vulnerabilidad, para implementar un programa de tutoría entre iguales con niños de 30 de primaria. Esta red se constituye como una comunidad profesional de aprendizaje, donde el proceso de formación del profesorado recibido para desarrollar la tutoría entre iguales en el aula, la reflexión colaborativa sobre la práctica educativa, así como los resultados de aprendizaje obtenidos por los estudiantes, ayudan a producir tensiones y promover cambios conceptuales en el profesorado, sobre la colaboración docente y cooperación entre estudiantes. A través de un estudio mixto, se mide de forma cuasiexperimental, a manera de pre-test y post test, los niveles de comprensión y otras habilidades lectoras de 134 alumnos que participan en el programa de tutoría, y a través de un estudio cualitativo se indaga las representaciones iniciales y finales de los docentes en concordancia con la implementación en el aula del programa. Los resultados indican que las mejoras del alumnado, así como el trabajo colaborativo entre profesores, han contribuido a promover un cambio en relación a las concepciones docentes acerca de la necesidad de colaborar y cooperar para alcanzar los objetivos educativos.

Palabras clave: Modificación representacional. Comunidades de aprendizaje. Colaboración docente. Tutoría entre iguales.

\begin{abstract}
This article presents a thorough review of literature about potentialities of collaboration between teachers in professional development, problems resolutions, and educational practice feeding, creating spaces for reflection that to prepare a change of approaches, representations and practices. Subsequently, this article also describes a collaboration between 13 teachers from 5 schools, in a high vulnerability Chilean context (Región de la Araucanía, Chile), in order to implement a peer tutoring program with children from 3rd grade of elementary school in scope of the investigation of project. This network is constituted as a professional learning community where the formation process tutoring in the classroom for teachers, the collaborative reflexion about the educational practice, as well as the learning outcomes obtained by the students help to produce tensions and promote conceptual changes in the teachers in relation to teacher collaboration and cooperation between students. Through a mixed study, the levels of comprehension and other reading abilities of 134 students who participate in the program were measured in a quasi-experimental, as pre-test and posttest. Also, through a qualitative study, the initial and final representations of the teacher, in correspondence with the implementation of the program in the classroom, was enquired into. The results indicate that the improvement in the student body as well as the collaborative work between teachers, have contributed to promote a change in relation to the teacher's conceptions about the need to collaborate and cooperate to achieve the educational objectives.
\end{abstract}

Keywords: Representational change. Learning communities. Teacher collaboration. Tutoring between equals.

Resumo: O artigo apresenta uma revisão exaustiva da literatura sobre as potencialidades da colaboração entre professores no desenvolvimento pessoal, na resolução de problemas e na retro-alimentação das práticas educativas, criando espaços de reflexão que preparam uma mudança de enfoques, representações e práticas. Posteriormente,

Artigo recebido em setembro de 2017
Aprovado em novembro de 2017

Cad. Pesq., São Luís, v. 24, n. 3, set./dez. 2017 
o artigo descreve uma rede de colaboração entre 13 professores de 5 escolas, num contexto chileno (Região de Araucanía, Chile) de grande vulnerabilidade, para implementar um programa de tutoria entre iguais com crianças de $3^{\circ}$ séries do primário no âmbito de um projeto de investigação. Esta rede se constitui como uma comunidade profissional de aprendizagem, onde o processo de formação do corpo docente recebido para desenvolver a tutoria entre pares em aula, a reflexão colaborativa sobre a prática educativa, assim como, os resultados de aprendizagem obtidos pelos estudantes, ajudem a produzir tensões e promovam mudanças conceptuais nos professores sobre a colaboração docente e a cooperação entre estudantes. Através de um estudo misto, mediu-se de forma quasi-experimental, de tipo pré-teste e pós-teste, os níveis de compreensão e outras habilidades de leitura de 134 alunos que participaram do programa de tutoria, e, através de um estudo qualitativo indagou-se sobre as representações iniciais e finais dos docentes de acordo com a implementação na aula do programa. Os resultados indicam que as melhorias nos alunos, tal como o trabalho colaborativo entre professores, contribuíram para uma mudança nas concepções docentes acerca da necessidade de colaborar e cooperar para alcançar os objetivos educativos.

Palavras-chave: Modificação representacional. Comunidades de aprendizagem. Colaboração docente. Tutoria entre iguais.

\section{INTRODUCCIÓN}

El presente artículo expone, en primer lugar, los antecedentes teóricos de las líneas fundamentales que sustentan la investigación, referida a colaboración docente y su incidencia en la conformación de las comunidades de práctica profesional, para posteriormente retomar el constructo de representaciones mentales y como se puede producir su cambio conceptual a través de los procesos implicados en dinámicas de colaboración docente y aprendizaje con otros. Ello da origen a los objetivos del estudio, la metodología de carácter mixto escogida, instrumentos y procedimientos implementados, para levantar los resultados referidos a los efectos de la tutoría entre iguales en los estudiantes, y las representaciones de los profesores en torno a la colaboración y cooperación. Las conclusiones relevan la importancia de la promoción de trasnformación de prácticas docentes a través de los procesos seguidos en la investigación y que repercuten en sus enfoques, perspectivas y representaciones sobre el proceso de enseñanza-aprendizaje.

En este estudio estamos de acuerdo en que para avanzar hacia la construcción de centros educativos que se conciban como comunidades de enseñanza y aprendizaje, aprovechando los recursos naturales aportados por sus integrantes, transformándolos en un motor de aprendizaje, se debe promover la cooperación, colaboración y aprendizaje entre los agentes educativos, especialmente entre los profesores. Se ha evidenciado en variadas investigaciones que la adopción de objetivos comunes, intercambio de experiencias y construcción de significados compartidos entre profesores (WEICK, 1995) tienen un impacto directo en el éxito de los estudiantes (LITTLE et al., 2003; BERRY; JOHNSON; MONTGOMERY, 2005; VESCIO; ROSS; ADAMS, 2008).

Existen muchos estudios que muestran cómo la colaboración entre profesores puede ayudarles a ellos mismos a generar conflictos cognitivos para resolver problemas, mejorar su práctica educativa, reflexionar con la retroalimentación y crítica de otros, plantear puntos de vista alternativos y desarrollar actitudes más positivas hacia la enseñanza (BROWNELL et al., 1997; SHACHAR; SHMUELEVITZ, 1997; BUYSSE; SPARKMAN; WESLEY, 2003; DE DREU; WEINGART, 2003). Por eso, es relevante en este estudio revisar, además, cómo ello repercute en un cambio de representaciones, cuando los profesionales se ven involucrados en prácticas de carácter constructivistas orientadas a la mejora de su quehacer y el aprendizaje de sus estudiantes, construyendo comunidades de aprendizaje que colaboran para alcanzar los objetivos educativos.

A continuación, se presentan los fundamentos de los ejes conceptuales que sustentan el presente estudio.

\section{COLABORACIÓN DOCENTE}

Si bien asumir un enfoque colaborativo de trabajo entre los profesores, creando comunidades de práctica profesional, es prioridad y política de muchos sistemas educativos, su puesta en práctica es compleja. Según Goddard, Goddard y Tschannen-Moran (2007), las dificultades se deben a que en la formación universitaria no se enseña deliberadamente, como competencia, la colaboración entre profesores; ni tampoco, durante su vida profesional, los profesores reciben un apoyo sustancial de sus colegas o administradores para retroalimentar su trabajo. 
Sin embargo, se ha evidenciado que las relaciones de colaboración entre docentes constituyen un factor importante para el desarrollo profesional y su aprendizaje (ERICKSON et al., 2005). Para que esta colaboración sea exitosa, debe poseer un sentido compartido, de interdependencia en el logro de resultados previstos, basada en la comunicación abierta, pero enfocada en los propósitos si se quiere construir significados individuales y comprensión mutua, produciéndose solo cuando se alcanzan las condiciones de colaboración en los entornos de aprendizaje (GARRISON, 2016).

Se destacan que estas condiciones no están exentas de complejidades, y por lo demás se distinguen de otras prácticas que involucran comunicación mutua e interdependencia, como es la cooperación. Se hace necesario distinguir ambas debido a que se encuentran involucradas en este estudio. Aunque sin un acuerdo completo, la literatura científica tiende a plantear que la colaboración sería un término más general con una interacción menos estructurada entre sus miembros, más espontánea, simétrica y flexible, mientras que el aprendizaje cooperativo requiere de una estructuración e intervenciones técnicas para vehiculizar las interacciones entre sus miembros y contribuir a la emergencia de las condiciones que garantizarían su éxito (MILLIS; COTTEL, 1998; O'DONNELL; KING, 1999; KNESER; PLOETZER, 2001).

Estamos de acuerdo en entender que la organización dentro de los grupos, para su transformación en equipos de trabajo, es necesaria para promover la cooperación, con lo cual discrepamos de entender la colaboración como la forma más sencilla de trabajar en equipo, ya que, evidentemente, si estas condiciones no emergen espontáneamente, el objetivo a alcanzar será más complejo aún. En este sentido, entenderemos la colaboración como una modalidad quizás aún más compleja que la cooperación, dependiendo mucho de la capacidad de sus miembros, el compromiso y la identificación con los objetivos finales, propia de contextos más informales o profesionales (TOPPING et al., 2017).

En un escenario $u$ otro, lo que parece relevante es la interacción entre las actividades de aprendizaje individuales y las de colaboración en las que se participa, ya que ponen en juego perspectivas divergentes y construcción compartida de conocimientos y significados (PUNTAMBEKAR, 2006; STAHL, 2002). Situar el aprendizaje en un contexto social puede potenciar la adopción de estrategias, respuestas a dificultades, toma de decisiones, retroalimentación, proponiéndose un nuevo accionar frente a problemas habituales en los centros educativos. Para que ello pueda concretarse, estas interacciones no pueden ser aisladas y los miembros de un grupo deben constituirse con objetivos compartidos, metas y compromisos que les permitirá transformar esa agrupación en comunidades profesionales de aprendizaje (BEREITER; SCARDAMALIA, 1993; BEREITER, 2002; LITTLE, 2002).

La relevancia de la creación de estas comunidades fundamentadas en la colaboración, según las investigaciones (GARRISON, 2016; DANA; YENDOL-SILVA, 2003), vendría dada por sus participantes y las interacciones (en forma de diálogo y actividad conjunta), que permitiría crear significados compartidos acerca de la práctica, a partir de las inquietudes y experiencias personales, dando respaldo a los maestros para realizar actuaciones con más seguridad y confianza. Esto se traduciría como espacios donde los docentes pueden crear, compartir, plantearse desafíos y enriquecer su repertorio de prácticas a través de una formación profesional basada en la colaboración con otros pares, lo cual no es una tarea simple, dentro de la cultura escolar tradicional. Las evidencias dan cuenta que las redes de maestros y las discusiones generadas en su interior son contextos poderosos para mejorar las prácticas y producir nuevas tensiones acerca de sus representaciones, por ejemplo en la experiencia de desarrollo profesional (ERICKSON et al., 2005).

Cuando se examinan los cambios en las prácticas de los maestros que participan en comunidades profesionales de aprendizaje, el profesorado muestra percepciones positivas y valiosas sobre sus propias prácticas de enseñanza y aprendizaje de sus estudiantes (VESCIO; ROSS; ADAMS, 2008). En este sentido, existen evidencias de su repercusión positiva en el aprendizaje del alumnado (GODDARD; GODDARD; TSCHANNEN-MORAN, 2007), reportando beneficios en alfabetización, lectura, escritura, fortalecimiento de valores o compromiso con su aprendizaje (PHILLIPS, 2003; STRAHAN, 2003; HOLLINS et al., 2004; BERRY; JOHNSON; MONTGOMERY, 2005).

Así mismo, existen características que identifican la creación a una comunidad profesional de aprendizaje y que la distinguen de otras instancias de colaboración más informales. Estas características, según Vescio, Ross e Adams (2008), son el desarrollo de valores y normas compartidas; una postura o enfoque unificado sobre el aprendizaje de los estudiantes, lo que 
asegurará su éxito y alcance de metas; y un diálogo reflexivo que conllevará a asumir posturas, cuestionar ideologías y representaciones sobre temáticas educativas. Todo ello permitiría promover y sostener el aprendizaje de los miembros de una comunidad, con el objetivo de mejorar sus prácticas en virtud del aprendizaje de sus estudiantes.

La participación en una comunidad de aprendizaje profesional parece tener potencial para promover en los docentes el desarrollo, fortalecimiento o modificación de prácticas vinculadas con su quehacer educativo y con ello, posiblemente, sus representaciones, a través del diálogo reflexivo y crítico que los llevaría a cuestionar sus posicionamientos para mejorar la enseñanza y aprendizaje propios, y de sus estudiantes.

\section{REPRESENTACIONES Y SU TRANSFORMACIÓN}

Para incorporar un enfoque colaborativo de trabajo y alcanzar la conformación de comunidades profesionales de aprendizaje, es necesario un cambio conceptual en las representaciones del propio profesorado (PÉREZ-ECHEVERRÍA et al., 2001). En este sentido, Pozo et al. (2006) señalan que las representaciones se constituyen como verdaderas teorías implícitas, que poseerían, por una parte, una naturaleza implícita y, por otra, una naturaleza teórica (representaciones organizadas y cohesionadas por ciertos principios). Su modificación implicaría un cambio en los principios en que se fundamenta el sujeto, lo cual no es tarea fácil (LIMÓN; MASON, 2002; ATKINSON; CLAXTON, 2002; SCHNOTZ; VOSNIADOU; CARRETERO, 1999; SCHÖN, 1987). Por tanto, para modificar de forma efectiva dichas teorías implícitas,es necesario que los docentes participen en una inducción explícita a través de una formación con objetivos planteados para ello (POZO et al., 2006).

Por lo tanto, para que se produzca el cambio conceptual docente hacía enfoques colaborativos, será necesario crear espacios de reflexión que cuenten con personas más expertas, para poder no sólo explicitar puntos de vista, sino aportar concepciones alternativas (HARGREAVES; FULLAN, 2000); utilizar instrumentos epistémicos (diarios, análisis de casos, revisiones de grabaciones 0 prácticas compartidas) (JAY; JOHNSON, 2002); y combinar el análisis de la propia práctica docente con la de otros profesores (MCINTYRE, 1993); o con datos que propicien la reflexión (SANTOS GUERRA, 2001). Uno de estos datos proviene del efecto que la práctica docente tiene sobre el propio aprendizaje de los estudiantes que atiende el profesor, del grado en que los estudiantes desarrollan la competencia (aprenden) y del grado en que la ayuda del profesor es efectiva (enseña) (HUBBER; TYTLER; HASLAM, 2010).

Entendemos entonces que la tensión de las representaciones gracias a la reflexión con otros y a través de la guía de un experto podría promover un cambio conceptual, sobre todo cuando la práctica educativa se ve sujeta y enriquecida a través de un análisis crítico, que permite al docente verse a si mismo como enseñante y aprendiz de nuevas estrategias, propuestas y alternativas que tengan repercusión directa en el proceso que desarrollan sus estudiantes.

\section{CREACIÓN DE COMUNIDADES PROFESIONALES DE APRENDIZAJE A TRAVÉS DE UN PROGRAMA DE TUTORÍA ENTRE IGUALES}

El estudio plantea una innovación basada en un programa de aprendizaje cooperativo, implementando la tutoría entre iguales (DURAN et al., 2011), con estudiantes de primaria de contextos socio-educativos vulnerables, donde los profesores a cargo de los niveles son invitados a conformar una red para constituirse en una comunidad profesional de aprendizaje.

En primer lugar, durante un semestre, los profesores son formados por un experto en prácticas constructivistas y, específicamente, en métodos de aprendizaje cooperativo, teniendo la oportunidad de reflexionar, cuestionar, analizar literatura y contrastarla con su realidad educativa, mientras implementan prácticas de aprendizaje cooperativo en colaboración con otros docentes. Tras la formación inicial, durante el siguiente semestre, los docentes son los responsables de implementar en sus aulas un programa de tutoría entre iguales en comprensión lectora, donde los estudiantes asumen roles de tutores y tutorados, concibiendose el aula como una comunidad que aprende a partir de las diferencias. Paralelamente, los docentes siguen siendo parte de la red (comunidad de aprendizaje), reflexionando conjuntamente sobre la puesta en práctica del programa en el aula y el aprendizaje entre profesores, como medio para favorecer un cambio conceptual que les permita disponer de representaciones adecuadas para incorporar la tutoría entre iguales en los repertorios 
metodológicos de sus centros educativos (THOUSAND; VILLA; NEVIN, 2006; MCDUFFIE; MASTROPIERI, SCRUGGS, 2009).

A partir de esta práctica educativa, el estudio que se presenta plantea los siguientes propósitos generales: conocer los efectos de la implementación de un programa de tutoría entre iguales con estudiantes de $3^{\circ}$ primaria, en las variables de dominio lector (velocidad lectora y calidad de lectura) y comprensión lectora, respecto a un grupo comparación de referencia, y por otra parte, determinar si hubo modificación representacional en los docentes en torno a la colaboración docente y la cooperación entre estudiantes, tras participar y constituirse como una comunidad profesional de aprendizaje.

El carácter mixto de la investigación, plantea una hipótesis (para el estudio cuantitativo) y una pregunta (para el cualitativo). Hipótesis: los estudiantes de $3^{\circ}$ de primaria que participan en la tutoría entre iguales alcanzarán diferencias estadísticamente significativas entre sus mediciones pre test y post test, en comprensión lectora y dominio lector, mientras que no lo harán los del grupo comparación. Y por otra parte, la pregunta de investigación sugiere indagar en la posible Pregunta: ¿Se produce una modificación representacional hacía la colaboración (entre docentes y entre estudiantes) propiciada por el trabajo en red de profesores para la implementación de la tutoría entre iguales, tras conocer el impacto en el aprendizaje de sus estudiantes?

\section{METODOLOGÍA}

El estudio responde a un diseño mixto de investigación, ya que esta mutualidad (cualitativa y cuantitativa) aporta una multiplicidad rica que permite responder, implicando diversos procedimientos, a los propósitos de investigación (CRESWELL, 2015). El estudio combina por una parte un estudio cuasi-experimental de tipo pre-test post-test con grupo de comparación para conocer la posible evolución de los estudiantes en sus habilidades de lectura y comprensión tras participar en un programa de tutoría entre iguales, implementado por sus profesores. Por otro lado, se consideró un estudio cualitativo de tipo descriptivo para explorar las representaciones de los docentes, previas a implementar la tutoría entre iguales y tras finalizar la experiencia.

Los participantes del estudio, se distinguen dos grupos que se presentan a continuación:

Grupo de Intervención: Se constituye por 5 escuelas de enseñanza primaria situadas en contextos socio-educativos vulnerables de la región de la Araucanía, Chile. Tanto sus directivos como profesores accedieron a participar voluntariamente en la experiencia de innovación, implicando también a los estudiantes. Participaron 13 profesores especialistas en Lenguaje o docentes de apoyo al aula de Educación Especial, responsables de formar parte de la red de trabajo docente e implementar el programa de tutoría. Durante la implementación, formaron parejas o tríos de trabajo entre docentes del mismo centro, para el desarrollo de la experiencia en el aula.

También participaron 134 estudiantes de $3^{\circ}$ de primaria (56 niños y 78 niñas), de los 5 centros educativos, cuyas edades fluctuaban entre los 8-9 años. Todos ellos desarrollaron el programa de tutoría por un semestre, conformado parejas de trabajo establecidas por el profesor y constituidas por tutor y tutorado. Durante 1 hora, dos veces por semana, leían en pareja y resolvían actividades de comprensión lectora preparadas por sus profesores.

Grupo de Comparación: conformado por 91 estudiantes de $3^{\circ}$ de primaria (47 niños y 44 niñas), de 4 escuelas de similares características socio-culturales del grupo de intervención. Ninguna de las escuelas de este grupo participó de la red de trabajo docente ni tampoco los estudiantes formaron parte del desarrollo del programa de tutoría entre iguales.

Por su parte, los instrumentos y técnicas de recogida de datos fueron las siguientes, para cada estudio:

a) Estudio Cuantitativo

Comprensión Lectora: CL-PT, Prueba de comprensión lectora y producción de textos (MEDINA; GAJARDO, 2012), de $1^{\circ}$ a $4^{\circ}$ básico. La prueba utilizada, de $3^{\circ}$ básico, evalúa comprensión lectora, producción de textos y manejo de la lengua y está, compuesta por 16 ítems. En el área de comprensión lectora, especialmente evaluada para nuestro estudio, se consideró estructura textual, comprensión literal, comprensión inferencial, comprensión crítica, reorganización de la información y comprensión metacognitiva. 
Dominio Lector: Se utilizaron las Pruebas Fundar (MARCHANT et al., 2004), para alumnos de enseñanza básica, específicamente para $3^{\circ}$ primaria. Combina la evaluación de la velocidad lectora con la calidad de lectura, a través de un texto leído en voz alta de forma individual, acompañado por una pauta que permite registrar los errores, el tiempo y la calidad de lectura. Este instrumento fue estudiado y validado en una aplicación experimental por sus autores (MARCHANT et al., 2000).

\section{b) Estudio Cualitativo}

Grupos Focales: Se realizaron 2 grupos focales con todos los profesores participantes, uno previo y otro al finalizar el estudio, para explorar la valoración otorgada a la creación de comunidades de aprendizaje para potenciar el aprendizaje colaborativo entre docentes, y también la cooperación entre estudiantes a través de la tutoría entre iguales. El instrumento de 12 ítems fue validado por un grupo de jueces expertos en el tema.

Entrevista semi-estructurada: Realizada a una muestra de 6 profesores, previo y al finalizar el estudio, con el objeto de complementar la información levantada en los grupos focales. El instrumento de 17 ítems -construido sobre los ejes de aprendizaje colaborativo entre docentes; tutoría entre iguales entre estudiantes; estrategias e implementación de actividades de comprensión lectora y dominio lector- fue validado por un grupo de jueces expertos en el tema.

Respecto a los procedimientos de investigación, durante el primer semestre se conforma una red de trabajo con los profesores participantes y responsables de la implementación de la tutoría entre iguales en el aula. Se realiza una exploración inicial de las representaciones explícitas acerca de la colaboración docente y el aprendizaje cooperativo entre estudiantes a través de entrevista y grupos focales.

Luego los docentes recibieron formación fundamentada en los principios constructivistas del aprendizaje cooperativo y sus diversos métodos, llevando a la práctica en sus aulas algunas experiencias y planificando la puesta en marcha del programa de tutoría entre iguales en el ámbito de la lectura que se desarrollaría el segundo semestre, incluyendo organización de sesiones, creación de parejas, calendarización de las lecturas y preparación de los materiales de lectura. Además, se realizó la evaluación inicial de comprensión lectora y dominio lector a los estudiantes de los centros educativos implicados y a un grupo de comparación.

En el segundo semestre, los profesores implementaron 25 sesiones de tutoría entre iguales en sus aulas, dos veces a la semana, por un tiempo de dos horas semanales. En el transcurso del semestre la red de profesores se reunieron cada 15 días para socializar con el experto la experiencia, reflexionar acerca de las problemáticas y soluciones. lo cual permitió tensionar las creencias que poseían acerca de las temáticas centrales del estudio.

Al finalizar se evaluaron las variables de comprensión lectora y dominio lector en los estudiantes, realizándose también una exploración final de representaciones con los docentes participantes.

En cuanto al estudio cuantitativo, el análisis de los datos contempló una prueba t-student para muestras relacionadas en función de pre test y post test, respecto a las variables dependientes (comprensión lectora y dominio lector), tanto del grupo intervención como comparación. Se utilizó SPPS 17.0.

Respecto al estudio cualitativo, tras la triangulación metodológica (FLICK, 2004), se procedió al análisis y codificación de los testimonios de los docentes, estableciéndose Dimensiones integradoras, y categorías, para llegar a analizar el "mensaje", considerado la unidad donde se sitúa la esencia del significado atribuido por los docentes a sus representaciones. El análisis se realizó con apoyo de Atlas ti 7.0.

\section{RESULTADOS}

Los resultados se presentan a continuación en función de los propósito del estudio.

\subsection{Efectos de la tutoría entre iguales en comprensión y dominio lector en los estudiantes}

Para la variable comprensión lectora, respecto al grupo de intervención las mediciones indican que el grupo de estudiantes presentó diferencias estadísticamente significativas entre las medidas iniciales y finales $(t(133)=-6.04, p<.01)$, tras participar de la implementación del programa fundamentado en la tutoría entre iguales (ver tabla 1). Por su parte, el grupo de comparación no 
evidenció diferencias significativas entre ambas mediciones $(t(90)=-1.89, p>.06)$ en la variable comprensión lectora.

En relación al dominio lector, el grupo de intervención mostró también avances estadísticamente significativos $(t(133)=-6.80, p<.01)$, al igual que el grupo de comparación $(t(90)=-3.29, p<.01)$ (ver tabla 1).

Tabla 1 - Resultados comprensión y dominio lector, grupo de intervención y comparación

\begin{tabular}{|c|c|c|c|c|c|c|c|c|c|}
\hline Variable & Grupo & $\mathrm{N}$ & M Pre-test & SD & M Post-test & SD & df & $t$ & $\mathrm{p}$ \\
\hline \multirow{2}{*}{ Comprensión Lectora } & Intervención & 134 & 19.83 & 6.39 & 23.39 & 8.03 & 133 & -6.04 & .00 \\
\hline & Comparación & 91 & 19.86 & 8.10 & 21.26 & 8.61 & 90 & -1.89 & .06 \\
\hline \multirow{2}{*}{$\begin{array}{l}\text { Dominio } \\
\text { Lector }\end{array}$} & Intervención & 134 & 85.25 & 26.03 & 96.40 & 28.16 & 133 & -6.80 & .00 \\
\hline & Comparación & 91 & 85.74 & 32.01 & 91.36 & 30.78 & 90 & -3.29 & .00 \\
\hline
\end{tabular}

Fuente: Elaborado por los autores.

Estos resultados permiten pensar que el profesorado participante ha podido constatar que sus alumnos mejoraban, en ambos factores, no sólo en dominio, sino también en comprensión, durante el proceso de puesta en práctica de la innovación basada en la tutoría ente iguales.

\subsection{Representaciones de los profesores en torno a la colaboración docente y la cooperación entre estudiantes}

A continuación, se presentan los hallazgos referidos a las representaciones iniciales y finales presentadas por los docentes participantes de la red, acerca de la colaboración docente y tutoría entre iguales.

\subsection{Representaciones de los profesores en relación a la colaboración docente}

Las categorías de análisis identificadas en esta dimensión son:

a) Colaboración docente en el interior del centro educativo-aula

En las representaciones explícitas iniciales, se dieron a conocer resistencias docentes frente a prácticas colaborativas y de co-docencia con otros profesores, como se evidencia en los siguientes testimonios entregados por los participantes:

"Es muy complejo y difícil encontrar los espacios para poder compartir lo que yo hago con mis colegas, incluso de ciclo o de la misma asignatura. No hay espacios establecidos en la escuela para ello". (Testimonio profesor 2, entrevista inicial).

"El día a día no permite que tengamos espacios donde podamos poner en común lo que hace cada uno en su aula, además no sé si todos estarían dispuestos a compartir y abrirse a que los critiquen u opinen sobre la forma en que desarrollan su clase o abordar determinados casos, por ejemplo de niños con necesidades educativas. En ese sentido, yo creo que aún hay muchas resistencias". (Testimonio profesor 5 , grupo focal inicial).

Se desprende de los testimonios iniciales, una carencia a nivel institucional de prácticas compartidas, resistencias que podrían deberse a las creencias que poseen los equipos directivos y docentes acerca de compartir con otros la enseñanza, el temor a recibir críticas sobre prácticas en el aula, y la falta de tiempo y espacios formales e institucionalizados en los centros educativos para realizar posibles colaboraciones con otros docentes. También se identifican los programas de Fomento de la Lectura (Iniciativas del gobierno) como instancias que no propician la colaboración o co-docencia con otros docentes, y que más bien potencian el trabajo individualizado de los docentes con sus niños, debido a la estructuración y rigidez del formato de los materiales y del trabajo inherente de este tipo de programa.

Respecto a los facilitadores en este ámbito, se destaca como facilitador en algunos centros educativos, el contar con un programa de integración donde la educadora diferencial participa activamente de las clases del profesor de asignatura, apoyando y coordinando estrategias para potenciar aprendizajes de los estudiantes con necesidades educativas especiales.

En cuanto a las representaciones explícitas finales de los docentes, tras haber participado de la experiencia de colaboración y de haber implementado el programa de tutoría entre iguales con sus estudiantes, estos declaran que visualizan como facilitadores la oportunidad de compartir con otros docentes de su centro educativo nuevas estrategias y comunicar las experiencias que estaban 
vivenciando en el aula como una instancia de retroalimentación de la práctica. Esto se sistematiza en las siguientes declaraciones de los profesores:

"Se nos ha dado la oportunidad de compartir en los consejos y reuniones técnicas lo que nosotros estamos haciendo, tanto a nivel de tutorías con los niños, como de la red con otros docentes de otras escuelas, con nuestros colegas. Eso te ayuda en tu labor en poder contar a los demás qué estrategias estás utilizando y cómo te está funcionando. Ellos te preguntan y también sugieren cómo podrían hacerlo ellos". (Testimonio profesor 2, grupo focal final).

También otro profesor señala: "Es beneficioso para mejorar nuestras prácticas, compartir estrategias, las reflexiones pedagógicas que podemos hacer de nuestras prácticas, reflexionar críticamente de lo que hacemos". (Testimonio profesor 5, entrevista final).

También se destaca que esto ha permitido que los docentes que participan en dupla o trío por cada centro educativo valoren los conocimientos que los otros poseen, su expertís y experiencia profesional de cada uno. Por ejemplo, los docentes señalan:

"Antes yo sabía que mis colegas trabajaban en $2^{\circ}$ y $3^{\circ}$ y que eran de lenguaje y matemáticas, pero más allá de lo que se trabaja en las reuniones técnicas, yo no había tenido la oportunidad de compartir con ellas aprendizajes. Por ejemplo, con la profesora de $2^{\circ}$ se hizo un excelente trabajo en las hojas de lectura, ella nos revisó, nos ayudó a seleccionar las lecturas adecuadas y luego entre los tres fuimos ajustando las preguntas. Fue un trabajo enriquecedor y que, si no hubiese sido por esta instancia, no hubiéramos tenido la oportunidad de compartir". (Testimonio profesor 8 , grupo focal final).

Los docentes también hacen mención a que participar en la comunidad de aprendizaje y compartir el trabajo desarrollado en sus centros educativos, les permitió propiciar mayores instancias de coordinación de otras actividades extra-programaticas que se vinculan al programa de lectura implementado (por ejemplo, olimpiadas de lectura), que involucran no solo a otros docentes del centro, sino también a otros establecimientos educativos. Se añade que la práctica docente se ha visto fortalecida, posibilitando las condiciones para implementar con esta experiencia prácticas de codocencia más amplias y no solo centradas en los programas de integración, donde puedan participar otros profesores. Ello evidencia la otorgación de una valoración positiva y apertura a prácticas educativas colaborativas que enriquecen la labor docente y el aprendizaje de los estudiantes.

Respecto a los obstaculizadores que se indagaron y que aún permanecen, hacen referencia a la implementación de programas gubernamentales de lectura, con una estructuración y rigidez que hace difícil la colaboración con otros docentes del centro para su implementación. Los profesores señalan que a pesar de ello buscan nuevas oportunidades, intentando incorporar las estrategias aprendidas.

b) Colaboración en la red con otros profesores (conformación comunidad profesional de aprendizaje)

Respecto a la colaboración en la red, las representaciones iniciales hacen referencia a la potencialidad de ejecutar un trabajo coordinado, ya que ello permitiría afrontar con confianza la innovación educativa. En este sentido, los docentes destacan como facilitadores el compromiso asumido por los directivos (administración) para concretar su participación en el programa; el plan de conformación de una red -comunidad profesional de aprendizaje entre profesores de distintos centros educativos-, lo cual aportó diversidad, experiencia, y fortaleza para afrontar el desafío de la implementación de un programa de tutoría entre iguales. Un ejemplo de ello se rescata en la siguiente declaración de un profesor:

"Yo lo encuentro super bueno, porque uno comparte con los colegas y como decia ella (otra profesora), compartir aquí las experiencias de cómo puede haber resultado el trabajo en su colegio y en su curso, si le resultó o no le resultó, porque... es una ventaja". (Testimonio profesor 6 , entrevista inicial).

Algunas de las resistencias u obstaculizadores identificados en el análisis de las representaciones docentes hacían referencia a experiencias previas poco satisfactorias, referidas a formaciones docentes que comprometían trabajo colaboratido y en red, pero que finalmente se transformaron en un trabajo aislado e individual de cada docente.

Las representaciones finales de los profesores confirmaron la oportunidad que les entregó la conformación de la comunidad de aprendizaje para promover la reflexión sobre la práctica docente; 
búsqueda de alternativas en grupo entre los docentes a las dificultades experimentadas en la puesta en práctica de la experiencia. Así lo destaca la siguiente declaración:

"Los profesores que participamos nos hemos visto fortalecidos en este aprender, que para mí también es algo nuevo que no conocía, pero se ha dado bastante rico en la preparación del material, en el trabajo de buscar estrategias. Yo creo que eso le hace falta a los consejos de profesores a las escuelas, este espacio de compartir, de conversar. Yo creo que enriquece. Nosotros quizás también somos profesores que estamos llamados a compartir o a replicar estas mismas experiencias con los colegas". (Testimonio profesor 7 , grupo focal final).

También los docentes destacan la transferencia de la metodología (tutoría entre iguales) que han podido realizar a otras asignaturas, como se expresa a continuación:

"Es bueno no solo en lenguaje, nosotros lo podemos aplicar en todas las asignaturas y empezar desde la base, desde pequeñitos, no con la dificultad que se le aplica a un tercero básico o un cuarto tal vez, pero ya comenzar a prepararlos para después". (Testimonio profesor 9, entrevista final).

Finalmente, hay un acuerdo de todos los docentes sobre el fortalecimiento de aprendizajes en lectura de manera transversal en el currículum por parte de sus estudiantes, como lo expresan algunos testimonios:

"Este trabajo ha generado muchas cosas positivas en la comprensión lectora. Los chicos estaban en un rango insuficiente y hoy creo que gracias al aporte de la lectura compartida han subido al nivel adecuado. Hoy día los niños infieren más, aportan más, creo que comprenden bastante lo que leen y eso lo veo porque, hay más colaboración y empatía". (Testimonio profesor 11, entrevista final).

"En la comprensión lectora yo encuentro que los chicos han avanzado un montón. El nivel de mi curso ha subido sí, me lo han reconocido los compañeros de trabajo, han notado cambio en todo sentido. Yo encuentro que este trabajo ha sido positivo, muy positivo". (Testimonio profesor 5, grupo focal final).

En este sentido, destacamos que el trabajo colaborativo gracias a la conformación de una comunidad profesional de aprendizaje ha tenido un impacto directo en los aprendizajes de los estudiantes. La socialización de las experiencias de tutoría entre iguales implementadas en cada colegio, se valora como una oportunidad de reflexión y retroalimentación de la práctica docente, una instancia de apoyo y de búsqueda de alternativas y soluciones que se presentan, que pueden ser a su vez resueltas por la comunidad de aprendizaje. Se identifica un cambio representacional, en el cual fue clave el acompañamiento en la red, seguimiento continuo, donde la retroalimentación de un experto, quien organizaba, mediaba y proponía la discusión en cada encuentro.

\subsection{Representaciones de los profesores respecto a la tutoría entre iguales}

Para esta dimensión las categorías de análisis levantadas fueron las siguientes:

c) Implementación en el aula

Se evidenció en la exploración inicial de representaciones una disposición positiva hacia la metodología, destacándose las creencias de los docentes acerca del fortalecimiento de aspectos socioemocionales en sus estudiantes, referidos a reconocimiento y valoración de las propias características entre pares, contribución a la convivencia escolar, clima de aula y desarrollo de valores como la solidaridad, respeto y cooperación entre pares, lo que vinculaban además con el control de la conducta y autorregulación de ésta (en casos de estudiantes disruptivos), desarrollo de la autoestima y diálogo constructivo entre pares. En este sentido, los docentes plantearon que:

"El aprender con otro a escuchar, a preguntarse, a ponerse en el lugar del otro además del propio aprendizaje de la lectura, les permite vivenciar valores que nosotros intentamos inculcar cada día: el compartir, cooperar por un objetivo común, ser solidario, ser empático". (Testimonio profesor 4, grupo focal final).

"El trabajo colaborativo permite a los estudiantes construir una convivencia armónica en el aula. Yo creo que además de los aprendizajes curriculares que traerá consigo, el tema del clima escolar, los valores, la sana convivencia es fundamental y nos ayudará en ese ámbito la tutoría". (Testimonio profesor 10, grupo focal final).

También los docentes plantearon que este tipo de prácticas permiten una ampliación de expectativas de aprendizaje, logros y vivencias en los estudiantes, vinculando al contexto de vulnerabilidad del cual provienen estos y las escasas oportunidades que se dan en el mismo contexto 
educativo de innovar, afrontando con nuevas estrategias el proceso de enseñanza-aprendizaje, sobretodo en el ámbito de la lectura.

Por otra parte, en esta indagación inicial de representaciones los docentes identificaron también obstaculizadores referidos a la baja calidad de las negociaciones que podrían establecer sus estudiantes, donde se podrían ver privilegiados acuerdos y respuestas unilaterales, lo cual no permitiría alcanzar los objetivos de la actividad cooperativa.

También los profesores destacaron algunas barreras propias del funcionamiento de la escuela y de la dinámica escolar, como el alto porcentaje de ausentismo escolar, lo cual podría interferir en la ejecución de las sesiones de lectura; el bajo compromiso y participación de las familias en el aprendizaje de sus hijos, lo cual podría repercutir en las tareas que los estudiantes debían asumir para que las sesiones de tutoría se desarrollasen con éxito; el gran número de estudiantes que comparten un aula, lo cual limitaría la organización física y podría dificultar el desarrollo optimo de la actividad; además de la carencia de otros espacios físicos (biblioteca, salas de apoyo) donde complementar el desarrollo de las sesiones.

Así, la exploración final de representaciones da cuenta que los docentes constataron que la tutoría entre iguales tuvo un impacto positivo en la convivencia escolar, clima de aula, desarrollo de habilidades sociales en los estudiantes (tanto tutores como tutorados), acuerdo y consenso de compromisos, normas para un desarrollo óptimo de la actividad, y valoración del otro compañero como fuente de apoyo a su aprendizaje.

También los profesores destacan como una predicción cumplida la ampliación de expectativas de los estudiantes, el desarrollo de la capacidad de asumir nuevos desafíos y vivencias con compromiso, repercutiendo en la construcción de la autoestima. Ello se declara en los siguientes testimonios:

"La autoestima creció bastante, tengo niños repitentes y que estaban muy mal y con muy malas notas, que hoy día tienen reconocimiento de sus compañeros y profesora; los que estaban bajos ahora son reconocidos como los mejores. Había una niña que era muy lenta y hoy día no. Hoy día pide tarea, pide reforzamiento, entonces la niña cambio bastante con esto, saben que es importante lo que están haciendo". (Testimonio profesor 9, grupo focal final).

"Muchos de mis estudiantes tenían temor a la respuesta errónea, ahora todos levantan su mano para responder, si se equivocan no importa, porque entendieron el error como fuente de aprendizaje". (Testimonio profesor 8 , grupo focal final).

Se declara también que sí se pudo dar la negociación y diálogo entre pares, que enriqueció posturas, respuestas y desarrollo de la actividad, lo cual permitió que emergieran los valores que ellos habían previsto inicialmente referidos a la solidaridad, cooperación y respeto. Al mismo tiempo, los docentes pusieron en marcha una estrategia innovadora que les permitió apoyar a sus estudiantes en el aprendizaje, resolución de dudas a través del seguimiento y monitoreo constante, desde un nuevo papel el de mediador.

En el caso de los estudiantes tutores, la mayoría recibieron el apoyo de sus familias para preparar la actividad de lectura en casa y luego replicarla con su compañero tutorado en el aula, tal como promovía el programa.

Finalmente, los docentes y sus directivos, buscaron estrategias alternativas para superar algunas de las limitaciones físicas con las que contaba el centro educativo para el óptimo desarrollo de la actividad. Si bien estos elementos fueron contemplados por la formadora en una etapa inicial, se pretendía que los mismos profesores y centros educativos ajustaran las condiciones de trabajo a las necesidades emergentes, para alcanzar el éxito de la actividad.

d) Prácticas docentes en comprensión lectora y dominio lector

En relación a la dimensión prácticas docentes en comprensión y dominio lector, inicialmente las representaciones dan cuenta de una concepción con fuerte influencia individualista y tradicional. Así, las prácticas indagadas y que se contemplaron en este estudio como obstaculizadoras hacen referencia a lecturas diarias con guías de comprensión lectora individual; lectura de libro y control individual mensual; actividades lectoras dirigidas por el profesor principalmente al grupo curso sin mayores adecuaciones a los ritmos de aprendizaje, intereses, motivación; desarrollo de actividades individuales a partir del libro de texto; fomento de la comprensión lectora a través de programas 
ministeriales; y evaluaciones de las prácticas antes descritas (escritas individuales y estandarizadas para conocer porcentaje de logro semestral y anual).

Como prácticas facilitadoras, en la línea de la tutoría entre iguales y de prácticas lectoras con un sentido constructivista, se destacan prácticas referidas a la socialización de lecturas con el grupo curso una vez resueltas de modo individual, y actividades de interrogación de texto dirigidas por el profesor al grupo curso.

La modificación representacional, al finalizar la intervención, indica que los profesores reconocen que pudieron combinar el trabajo en parejas o de pequeño grupo, con sus prácticas tradicionales habituales. Así, se complementó el trabajo con el libro de texto, desarrollo de guías de trabajo con socializaciones o resolución grupal o en parejas. Algunas de las prácticas lectoras individuales y lectura silenciosa, fueron reemplazadas por prácticas de lectura con audiencia y lectura conjunta, promovieron el gusto por la lectura en niños/as con antecedentes de fracaso escolar. También las evaluaciones de estos procesos sufrieron modificaciones y se combinó la evaluación individual con evaluaciones en pareja, a modo de co-evaluación de las prácticas lectoras y actividades de comprensión lectora.

Los docentes identificaron que algunos obstaculizadores de la práctica siguen siendo la implementación de programas de lectura estructurados e individualistas que se promueven desde el nivel central (gobierno); y la medición semestral y anual de los índices de comprensión lectora a través de pruebas estandarizadas.

\section{CONCLUSIONES}

El estudio aporta elementos a considerar para promover la transformación de prácticas docentes, que potencien el éxito y la participación de todo el alumnado (especialmente de aquellos en situación de vulnerabilidad). Se ha constatado las dificultades para promover un trabajo colaborativo entre docentes y también es reconocida la dificultad que tiene el aprendizaje cooperativo para llevarse a la práctica (SHARAN, 2010), incluyendo Latinoamérica y áreas de vulnerabilidad, como a la que hace referencia este trabajo.

La creación de esa red de profesores, pertenecientes a distintos centros educativos, pero que se constituyen en comunidad profesional de aprendizaje parece haber ayudado a operar cambios en la modificación representacional docente, tanto respecto a la propia colaboración docente como a la cooperación entre alumnos. La reflexión y trabajo constructivo con otros profesionales y expertos serían elementos nucleares para la que los profesores se vean dispuestos y apoyados para incorporar esas prácticas innovadoras, coincidiendo con las propuestas referenciadas en la introducción (VESCIO; ROSS; ADAMS, 2008). Los profesores declaran cambios debido a las oportunidades que han tenido de reflexionar, compartir dificultades y retos, en la puesta en práctica del programa de tutoría entre iguales con los alumnos, buscando apoyo y acompañamiento en la implementación de la innovación. En definitiva, se reconoce la colaboración docente como una forma de aprendizaje entre iguales -en este caso entre profesores.

En este sentido, los resultados apoyan desde la práctica los trabajos referenciados en la introducción del artículo, y están en línea con investigaciones en las que se crean comunidades de colaboración docente para la mejora de la práctica en un área curricular concreta (CHONG; KONG, 2012).

Además, los resultados positivos, especialmente en la comprensión lectora tras la implementación del programa, parecen haber tenido un impacto en el cambio de representaciones docentes, tal como explicitan los profesores. La constatación de dichas mejoras, a través de la observación directa durante el proceso, o a través de aportar los resultados en forma de datos, parece haber afectado positivamente la inducción explícita de la modificación representacional docente, en este caso enmarcada en la formación e implementación de un programa de tutoría entre iguales, en colaboración con otros docentes conformando comunidades profesionales de aprendizaje. Lo cual está en línea con los trabajos referenciados en los que se utilizan datos y observaciones sobre el impacto de la práctica docente en el aprendizaje del alumnado (GODDARD; GODDARD; TSCHANNEN-MORAN, 2007).

Finalmente, en un ámbito más práctico, quisiéramos destacar la potencialidad de generar formatos de mejora de práctica educativa a través de la colaboración docente, como el planteado, en la que se promueven deliberadamente tres tipos de aprendizaje entre iguales: entre los alumnos 
(en este caso a partir del programa de tutoría entre iguales); entre profesores (que llevan a la práctica el programa y reflexionan y aprenden unos de otros); y entre escuelas (que conforman dicha red y que, a su vez, permiten ofrecer apoyos y formas alternativas de resolver los retos que promueven aprendizaje entre instituciones). Este triple nivel de aprendizaje entre iguales (MIQUEL; DURAN, 2017) puede ayudar a entender la interacción con los demás -en forma de colaboración o cooperación- como una excelente fuente de aprendizaje y desarrollo profesional. 


\section{REFERÊNCIAS}

ATKINSON, T.; CLAXTON, G. El profesor intuitivo. Barcelona: Octaedro, 2002.

BEREITER, C. Education and mind in the knowledge age. Mahwah, NJ: Lawrence Erlbaum Associates, 2002.

BEREITER, C.; SCARDAMALIA, M. Surpassing our selves: an inquiry into the nature and implications of expertise. Chicago: Open Court, 1993.

BERRY, B.; JOHNSON, D.; MONTGOMERY, D. The power of teacher leadership. Educational Leadership, v. 62, n. 5, p. 56-60, 2005.

BROWNELL, M. et al. Teachers working together: what teacher educators and researchers should know. Teacher Education and Special Education, v. 20, n. 4, p. 340-359, 1997.

BUYSSE, V.; SPARKMAN, K.; WESLEY, P. Communities of practice: connecting what we know with what we do. Exceptional Children, v. 69, n. 3, p. 263277, 2003.

CHONG, W. H.; KONG, C. A. Teacher collaborative learning and teacher self-efficacy: the case of lesson study. The Journal of Experimental Education, v. 80 , n. 3, p. 263-283, 2012.

CRESWELL, J. A concise introduction to mixed methods research. California: Sage, 2015.

DANA, N. F.; YENDOL-SILVA, D. The reflective educator's guide to classroom research: learning to teach and teaching to learn through practitioner inquiry. Thousand Oaks: Sage, 2003.

DE DREU, C. K. W.; WEINGART, L. R. A contingency theory of task conflict and performance in groups and organizational teams. In: WEST, M. A.; TJOSVOLD, D.; SMITH, K. G. (Ed.). International handbook of organizational teamwork and cooperative working. West Sussex, UK: Wiley, 2003. p. 151-166.

DURAN, D. et al. Leemos en pareja: tutoría entre iguales para la competencia lectora. Barcelona: Horsori, 2011.

ERICKSON, G. et al. Collaborative teacher learning: findings from professional development projects. Teaching and Teacher Education, v. 21, n. 7, p. 787798, 2005.

GARRISON, R. Thinking collaboratively: learning in a community of inquiry. New York: Routledge, 2016.

GODDARD, Y.; GODDARD, R.; TSCHANNEN-MORAN, M. A theoretical and empirical investigation of teacher collaboration for school improvement and student achievement in public elementary schools. The Teachers College Record, v. 109, n. 4, p. 877896, 2007.

HARGREAVES, A.; FULLAN, M. Mentoring in the new millennium. Theory Into Practice, v. 39, n. 1, p. 50-57, 2000.

HOLLINS, E. et al. Promoting a self-sustaining learning community: investigating an internal model for teacher development. International Journal of Qualitative Studies in Education, v. 17, n. 2, p. 247-264, 2004.

HUBBER, P.; TYTLER, R.; HASLAM, F. Teaching and learning about force with a representational focus: pedagogy and teacher change. Research in Science Education, v. 40, n. 1, p. 5-28, 2010.

JAY, J.; JOHNSON, K. Capturing complexity: a typology of reflective practice for teacher education. Teaching and Teacher Education, v. 18, n. 1, p. 73-85, 2002.

KNESER, C.; PLOETZNER, R. Collaboration on the basis of complementary domain knowledge: observed dialogue structures and their relation to learning success. Learning and Instruction, v. 11, n. 1, p. 53-83, 2001.

LIMÓN, M.; MASON, L. (Ed.). Reconsidering conceptual change: issues in theory and practice. $\mathrm{Ne}-$ therlands: Springer, 2002.

LITTLE, J. W. Locating learning in teachers'communities of practice: opening up problems of analysis in records of everyday work. Teaching and Teacher Education, v. 18, n. 8, p. 917-946, 2002.

LITTLE, J. W. et al. Looking at student work for teacher learning, teacher community, and school reform. Phi Delta Kappan, v. 85, n. 3, p. 184-192, 2003.

MARCHANT, T. et al. Evaluación de dominio lector en alumnos de educación general básica. Santiago, Chile: [s.n.], 2000. (Serie Documentos Internos Fundar, n. 40).

MARCHANT, T. et al. Pruebas de dominio lector FUNDAR, para alumnos de enseñanza básica. Santiago, Chile: Universidad Católica de Chile, 2004.

MCDUFFIE, K.; MASTROPIERI, M.; SCRUGGS, T. Differential effects of peer tutoring in co-taught and non-co-taught classes: results for content learning and student-teacher interactions. Exceptional Children, v. 75, n. 4, p. 493-510, 2009. 
MCINTYRE, D. Theory, theorizing and reflection in initial teacher education. In: CALDERHEAD, K.; GATES, P. (Ed.). Conceptualizing reflection in teacher development. London: Falmer Press, 1993. p. 3952.

MEDINA, A.; GAJARDO, A. M. Pruebas de comprensión lectora y producción de textos: (CL-PT) Kinder- $4^{\circ}$ básico. Santiago, Chile: Universidad $\mathrm{Ca}-$ tólica de Chile, 2012.

MILLIS, B. J.; COTTELL, P. G. Cooperative learning for higher education faculty. Phoenix: Oryx Press, 1998.

MIQUEL, E.; DURAN, D. Peer learning network: implementing and sustaining cooperative learning by teacher collaboration. Journal of Education for Teaching, v. 43, n. 3, p. 349-360, 2017.

O'DONNELL, A.; KING, A. (Ed.). Cognitive perspectives on peer learning. New Jersey: Lawrence Erlbaum Associates, 1999.

PÉREZ-ECHEVERRÍA, M. del P. et al. En busca del constructivismo perdido: concepciones implícitas sobre el aprendizaje. Studies in Psychology, v. 22, n. 2, p. 155-173, 2001.

PHILLIPS, J. Powerful learning: creating learning communities in urban school reform. Journal of Curriculum and Supervision, v. 18, n. 3, p. 240-258, 2003.

POZO, J. I. et al. Nuevas formas de pensar la enseñanza y el aprendizaje. Barcelona: Graó, 2006.

PUNTAMBEKAR, S. Analysing collaborative interactions: divergence, shared understanding and constructions of knowledge. Computers and Education, v. 47, n. 3, p. 332-351, 2006.

SANTOS GUERRA, M. Á. La escuela que aprende. Madrid: Morata, 2001.
SHACHAR, H.; SHMUELEVITZ, H. Implementing cooperative learning, teacher collaboration and teachers' sense of efficacy in heterogeneous junior high schools. Contemporary Educational Psychology, v. 22, n. 1, p. 53-72, 1997.

SHARAN, Y. Cooperative learning for academic and social gains: valued pedagogy, problematic practice. European Journal of Education, v. 45, n. 2, p. 300313, 2010.

SCHNOTZ, W.; VOSNIADOU, S.; CARRETERO, M. New perspectives on conceptual change. London: Elsevier, 1999.

SCHÖN, D. Educating the reflective practitioner. San Francisco: Joseey Bass, 1987.

STAHL, G. Rediscovering CSCL. In: KOSCHMANN, R. H. T.; MIYAKE, N. (Ed.). CSCL 2: carrying forward the conversation. Mahwah, NJ: Erlbaum, 2002. p. 275-296.

STRAHAN, D. Promoting a collaborative professional culture in three elementary schools that have beaten the odds. The Elementary School Journal, v. 104, n. 2, p. 127-146, 2003.

THOUSAND, J.; VILLA, R.; NEVIN, A. The many faces of collaborative planning and teaching. Theory into Practice, v. 45, n. 3, p. 239-248, 2006.

TOPPING, K. et al. Effective peer learning: from principles to practical implementation. London; New York: Routledge, 2017.

VESCIO, V.; ROSS, D.; ADAMS, A. A review of research on the impact of professional learning communities on teaching practice and student learning. Teaching and Teacher Education, v. 24, n. 1, p. 80-91, Jan. 2008.

WEICK, K. Sensemaking in organizations. London, UK: Sage Publications, 1995. 